\title{
Current Status of Breast MRI -Clinical applications
}

Deepika Gunawardena ${ }^{1}$

${ }^{1}$ Department of Radiology, Royal Perth Hospital, Western Australia

\section{Abstract}

Breast MRI is a powerful imaging tool with an excellent sensitivity around 94-100\%, especially when double read, to detect cancers which are otherwise occult. However, lower specificity and high false positivity rates, demands histological confirmation of MRI only detected lesions to prevent possible over treatment. In order to maintain high standards and cost effectiveness of breast MRI, careful patient selection is as important as maintaining a meticulous technique or radiologist expertise. Breast MRI is useful at every phase of breast cancer management such as early detection, pre-operative staging, monitoring neo-adjuvant chemotherapy and identifying recurrences. This is a review on current best practice, for clinical applications of breast MRI.

Key Words: Breast, MRI,Screening

Copyright: Deepika Gunawardena

Correspondence: Deepika Gunawardena, dsguna@yahoo.com 


\section{Introduction}

Breast MRI is a powerful imaging tool with an excellent sensitivity around 94-100\%, especially when double read, to detect cancers which are otherwise occult. ${ }^{1}$ However, lower specificity and high false positivity rates, demands histological confirmation of MRI only detected lesions to prevent possible over treatment. ${ }^{2}$ In order to maintain high standards and cost effectiveness of breast MRI, careful patient selection is as important as maintaining a meticulous technique or radiologist expertise. ${ }^{3}$ Breast MRI is useful at every phase of breast cancer management such as early detection, pre-operative staging, monitoring neo-adjuvant chemotherapy and identifying recurrences. (Fig 1) This is a review on current best practice, for clinical applications of breast MRI.

\section{Fig.1. Indications for breast MRI}

1. High risk screening

2. Occult primary breast cancer

3. Problem solving breast MRI

4. Guidance for localization and biopsy

5. Pre- operative staging of breast cancer

6. Evaluation of response after neoadjuvant chemotherapy

7. Evaluation of breast prostheses

\section{High risk screening}

Mammography is the universally accepted screening modality to diagnose breast cancer in asymptomatic women, contributing to a significant reduction in mortality rates. Research on breast cancer has identified, certain groups in the population carrying higher than average life time risk for development of breast cancer. Unfortunately they are younger females and have reduced sensitivity to mammography due to denser breasts. Numerous studies around the world has consistently favour the use of breast MRI for screening this particular group with a life time risk over $20-25 \% .{ }^{3}$ Women with BRCA1 and BRCA2 gene mutations and their first degree relatives are an important group considered here. It is estimated that $50 \%$ of BRCA1 carriers develop the disease before the age of 50 . Chest irradiation for lymphoma in $2^{\text {nd }}$ or $3^{\text {rd }}$ decade, sufferers of inherited conditions like Lifraumeni and Cowdens disease and their first degree relatives will all benefit with breast MRI screening. Women carrying over 20\% lifetime risk for development breast cancer, on family history are also considered for screening with breast MRI. History of two $1^{\text {st }}$ or $2^{\text {nd }}$ degree relatives on the same side of the family with breast or ovarian cancer, when combined with an additional risk factor fulfill the required risk level. Additional risk factor could be any one of the following: a $3^{\text {rd }}$ relative with breast cancer, bilateral breast cancer in one of them, breast cancer diagnosed before the age of 40 years, male breast cancer or both breast and ovarian cancer in one. ${ }^{4}$

Extremely dense breasts, previous borderline lesions like lobular carcinoma in situ (LCIS), atypical lobular hyperplasia (ALH),atypical ductal hyperplasia (ADH) or life time risk of $15 \%$ are not yet considered for routine screening breast MRI. ${ }^{3}$

MRI screening starts around thirty years of age or 5 years earlier than the age at which the youngest family member was diagnosed with breast cancer, whichever comes earlier. Value of breast MRI will drop with clearing breast density as the age advances, however sensitivity of breast MRI is superior to mammography at any given age. A breast MRI sensitivity of $71-100 \%$ compared to mammographic sensitivity of $16-40 \%$ and MRI specificity of $81-99 \%$ compared to 
mammographic specificity of 93-99\% have been achieved in several small studies performed on these high risk women. ${ }^{3}$

\section{Occult primary breast cancer}

There is a small but challenging group of women presenting with axillary lymph adenopathy or distant metastatic disease suggesting a breast primary, with negative conventional imaging results. Breast MRI has been able to detect primary breast cancers in about $50 \%$ of them, sparing mastectomies in some. Rest of the women will have to proceed with either mastectomy or whole breast radio-therapy ${ }^{5}$ (Fig 2).
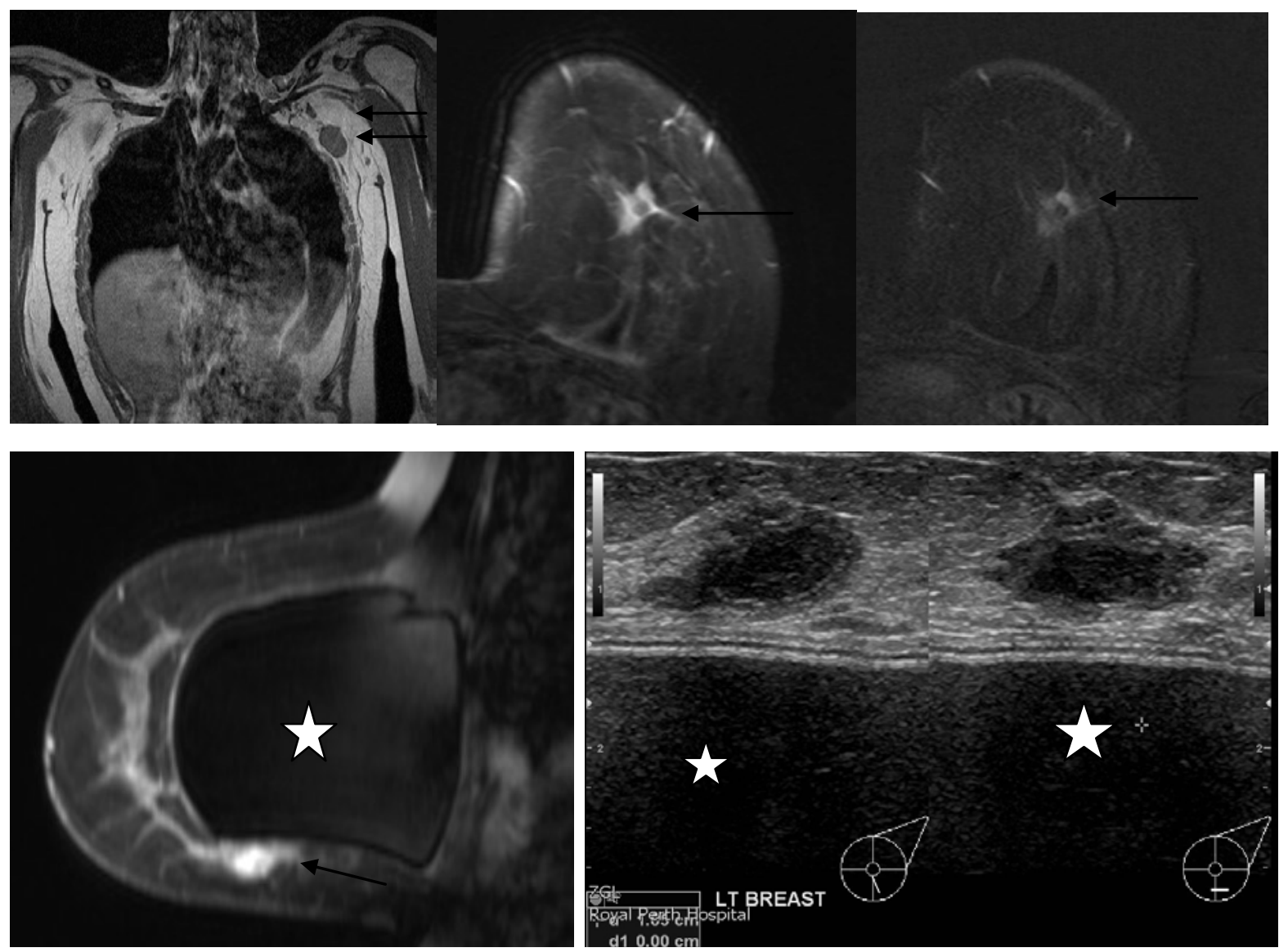

Figure 2. A 53 years old female presented with left axillary lymphadenopathy. Mammogram revealed dense breasts, bilateral breast prosthesis and axillary lymphadenopathy only. Breast MRI was performed for an occult primary. A coronal image on body coil shows left axillary lymphadenopathy. (A) Early post contrast subtraction image shows a small enhancing lesion of $15 \mathrm{~mm}$ with an irregular out line (B) Late contrast washout of the same lesion (C). Reconstructed sagittal image of left breast with fat saturation, shows the lesion along the inferior surface of the prosthesis. (D) $2^{\text {nd }}$ look left breast ultrasound scan and guided core biopsy confirmed the non palpable complex cystic primary breast carcinoma at 5 O`C position of left breast (E).

[Axillary lymphadenopathy $\rightarrow$ Enhancing lesion $\rightarrow$ Breast prosthesis $\hat{\sim}$ ] 


\section{Problem solving breast MRI}

MRI is an attractive problem solving tool for discrepancies in conventional imaging modalities and for sampling failures for pathological assessments. MRI has the greatest sensitivity of all imaging modalities and a negative MRI scan generally excludes malignancy except for cases with micro calcifications. ${ }^{3}$ Postoperative breast MRI is useful for women with positive excision margins on histology, following wide local excision. Four to six weeks after surgery is considered as optimal timing to assess residual disease. Interestingly, radiotherapy has no effect on accuracy of breast MRI. Suspected recurrence or screening after breast conservative therapy are two other occasions to use breast MRI confidently, as the specificity of MRI is higher at these instances than in a non- treated breast. Local recurrences usually occur within 5 years of index lesion and the risk of local recurrence is strongly related to the younger age at initial diagnosis. A $2^{\text {nd }}$ primary in the same breast typically results later than that. ${ }^{3}$

\section{Guidance for localization and biopsy}

When a new suspicious lesion is detected on MRI, a $2^{\text {nd }}$ look ultrasound scan and a review of mammograms are performed as a routine to plan a biopsy and marker clip insertion. When both these modalities fail, MRI guided biopsy is crucial, even though it is expensive, time consuming, technically challenging and need intravenous contrast administration. ${ }^{6}$ Alternatively short term follow up breast MRI, is recommended for small or less concerning abnormalities, in the absence of a known breast carcinoma

\section{Pre-operative staging of breast cancer}

Breast conservative surgery is the current trend for treatment of small tumors with a favorable lesion to breast volume ratio. Accurate pre-operative radiological quantification of the tumor is essential to avoid re-excision for positive excision margins on histology and to prevent early recurrences. Contrast enhanced breast MRI is proven to give lesion measurements closest to that on pathology, when compared to conventional imaging. MRI detects additional tumor focci in the same breast in $10-30 \%$ and synchronus contralateral breast cancers in $3-5 \%$ of breast cancer patients. ${ }^{3}$ Fischer et al has found a drop in local recurrence rate to $1.2 \%$ in the preoperative MRI group from 6.8\% recurrence rate in non pre-operative MRI group. ${ }^{7}$ Pre-operative staging breast MRI is still a controversial issue, as there is no proven reduction in reexcision rates and costs or improvement in breast cancer survival associated with it. However, dense breasts, poorly defined lesions or lesions close to chest wall on conventional imaging and histology of invasive lobular carcinoma are strong contenders for preoperative MRI staging. ${ }^{8}$

\section{Evaluation of response after neo-adjuvant chemotherapy}

MRI has shown to be superior to clinical and conventional imaging, in detecting tumor response to chemotherapy prior to surgery. This should preferably include a baseline MRI scan prior to treatment, a $2^{\text {nd }}$ MRI scan half way through the course of treatment and a $3^{\text {rd }}$ MRI scan after completion of chemotherapy. Response Evaluation Criteria in Solid Tumors (RECIST) criteria is used to evaluate the treatment response. RECIST criteria are described as a complete response (CR) when there is a completely vanished tumour. Partial response (PR) is over 30\% decrease in the sum of long axis measurements of all individual lesions. Progressive disease (PD) is over 25\% increase in the sum of long axis measurements of all individual lesions and stable disease (SD) is the remainder. Even after complete radiological response, 
resection of tumour bed is indicated as there is no proof for complete pathological response. ${ }^{3}$ A marker clip deployed at the center of the lesion prior to neo adjuvant chemotherapy, is used for hook-wire localization at a later date.

\section{Evaluation of breast prostheses}

Breast MRI with specific sequences has a sensitivity of $80-90 \%$ and specificity of $90 \%$ for diagnosis of implant complication. ${ }^{3} \mathrm{MRI}$ should only be used when there is a clinical suspicion of an implant problem which usually happens after about 10 years. Breast MRI with a tumor protocol can detect recurrent disease in a reconstructed breast without any compromise due to prosthesis. ${ }^{3}$

\section{Conclusion}

Breast MRI is a sophisticated adjunct to conventional imaging but not a substitute.

Breast MRI is not recommended for screening an average risk woman. Breast MRI should neither supplant a careful problem solving mammogram and ultrasound in a diagnostic setting nor use in lieu of a biopsy in the presence of a suspicious lesion. $^{9}$ In the absence of universally accepted guide lines, careful patient selection for breast MRI play a substantial role in maintaining high standards in breast cancer care.

\section{References}

1. Warren R, Pointan L, Thompson D (2005) Reading protocol for dynamic contrast-enhanced MR images of the breast: sensitivity and specificity analysis. Radiology. 236: 779-788. http://dx.doi.org/10.1148/radiol.2363040735
2. Kuhl K (2007) Current status of breast MRI imaging part 2 clinical applications. Radiology. 244:672-691 http://dx.doi.org/10.1148/radiol.2443051661

3. Mann RM, Kuhl CK, Kinkel K, Boetes C (2008) Breast MRI: Guidelines from European Society of Breast Imaging Eur Radiol 18:1307-1318 http://dx.doi.org/10.1007/s00330-008-0863-7

4. Eitan A, Orit C, Seruga D (2010) Assessing women at high risk of breast cancer: A review of risk assessment models. J Natl cancer inst.102(10):680-691 http://dx.doi.org/10.1093/jnci/djq088

5. Buchanan CL, Morris EA, Dorn PL (2005) Utility of breast magnetic resonance imaging in patients with occult primary breast cancer. Annals of surgical oncology 12(12): 1045-1053 http://dx.doi.org/10.1245/aso.2005.03.520

6. Park VY, Kim M, Kim E (2013) Second look US: How to find breast lesions with a suspicious MR imaging appearance. Radiographics 33:1361-1375 http://dx.doi.org/10.1148/rg.335125109

7. Fisher U, Zacharie O, Baum F, (2004) The influence of preoperative MRI of the breasts on recurrence rate in patients with breast cancer. Eur Radiol 14: 1725-1731.

http://dx.doi.org/10.1007/s00330-004-2351-z

8. Brasic N, Wisner D, Joe B (2013) Breast MR imaging for extent of disease assessment in patients with newly diagnosed breast cancer. Magn Reson Imaging Clin N Am. 21:519-532 http://dx.doi.org/10.1016/j.mric.2013.04.012

9. ACR Practice parameter for the performance of contrast-enhanced MRI of the breast. Amended 2014 (Resolution 39). 